\title{
ENERGY TRANSFER PROPERTIES AND MECHANISMS
}

\author{
TECHNICAL PROGRESS REPORT \\ (30 January 1992) \\ John R. Barker \\ Department of Atmospheric, Oceanic, and Space Sciences \\ $\mathrm{DOE} / \mathrm{ER} / 13442--6$ \\ DE92 011215 \\ The University of Michigan \\ Ann Arbor, Michigan 48109-2143
}

\section{Introduction}

Collisional energy transfer is the controlling factor in many nonequilibrium chemical systems. In combustion, both pyrolysis reactions and recombination reactions are largely controlled by energy transfer. In infrared laser-induced chemistry, collisional energy transfer is , often a limiting factor, because of degradation of the excited species population distributions. In shock-heated gases and other hostile environments, energy transfer plays a significant role. In atmospheric science, radical recombination reactions are controlled largely by energy transfer.

The overall objective of the research carried out under this program is to determine the principles of collisional energy transfer and use them in predictive models and theories. In order to accomplish this goal, energy transfer properties must be determined and then analyzed to discern the underlying principles involved.

In this laboratory, the experimental determination of energy transfer parameters is based on techniques that use physical properties to monitor the amount of energy in excited molecules. These techniques differ from chemical methods, based on unimolecular reaction studies, which are susceptible to interferences from complex chemical mechanisms and other complications. The physical methods have their own weaknesses and limitations, however, and much of our effort has been directed toward gaining a better understanding of these deficiencies. Two physical techniques have been proved to be particularly useful: time-resolved infrared fluorescence (IRF) and timedependent thermal lensing (TDTL). As described later, we will shortly begin work using resonance enhanced multiphoton ionization (REMPI) techniques to investigate energy transfer in bulbs and "half collisions" in free jets.

We also have been completing some calculational efforts to model formation of polycyclic aromatic hydrocarbons in stellar atmospheres. These calculations are natural applications of the

\section{MASTER}


detailed chemical schemes developed for soot formation in hydrocarbon flames. In other calculations, we are exploring the influence of spontaneous infrared emission on stabilization of excited adducts produced in radical-radical recombination reactions.

\section{Accomplishments and Plans}

The IRF technique continues to be a real workhorse. The work on benzene, toluene, and vibration-to-vibration (V-V) energy transfer to $\mathrm{CO}_{2}$ from benzene derivatives have been published. The experimental work on deactivation of benzene and toluene isotopomers has been completed and the manuscript is in preparation. All of this work tends to support an emerging general view of large molecule vibrational deactivation:

1. V-T and V-R energy transfer occurs with all colliders, probably due to short range repulsions on the potential energy surface.

2. V-V energy transfer takes place by long-range dipole-dipole interactions.

3. If the collision partner has a permanent dipole moment, V-R energy transfer is probably enhanced, due to coupling between the permanent dipole and the time-dependent transition dipole in the excited molecule.

4. Deuteration has little effert on vibrational deactivation, probably because most of the deactivation occurs by V-T/R transfer from the lowest frequency vibration mode (deuteration has little effect on the lowest frequencies).

5. Classical trajectory methods are inherently flawed. Even their approximate validity for simulations of large molecules has not been demonstrated and must be investigated.

In other IRF studies, we have obtained the time-resolved spectra of highly excited benzene as it is being deactivated. These spectra show three components, corresponding to $\Delta v=-1$ transitions originating from $v^{\prime}=1,2$, and 3 . The anharmonic shifts agree with literature values and the whole spectrum is shifted for highly excited benzene, compared to the unexcited molecule. The relative intensities of the components at $t=0$ agree very well with theory. At later times, the relative intensicies reflect the evolution of the population distribution of excited species. We are planning to exploit this property in order to determine more detailed information about the distribution as it evolves with time. The details depend on the energy transfer properties and we will use our Monte Carlo master equation computer code to simulate the experiments and find the energy transfer 
properties that reproduce the experiments. This work is just beginning, but we already have a paper in press that shows the astrophysical implications of the results.

In another experiment, we have used the TDTL technique to investigate highly excited $\mathrm{CS}_{2}$ deactivated by $\mathrm{Ar}, \mathrm{Kr}$, and $\mathrm{Xe}$. These results are in some ways similar to those we obtained for $\mathrm{NO}_{2}$. The data analysis is time-consuming and not yet complete, but we anticipate that the work will be complete in the first quarter of 1992.

We are preparing to carry out REMPI experiments in bulbs and in molecular free jets. The first experiments will use a pump-probe arrangement to investigate the rovibrational populations of collision partners in the deactivation of highly excited benzene. The first laser pulse $(248 \mathrm{~nm})$ will excite the benzene. After a selected time delay (time resolution of $\sim 20 \mathrm{~ns}$ ) the tunable dye laser pulse will be used to ionize the collision partner from selected states (REMPI). We expect that V-R energy transfer is very important and this technique will enable us to determine its magnitude, relative to V-V energy transfer, for example. This work will begin sometime this spring.

We are also installing a linear time of flight mass spectrometer in our pulsed molecular jet apparatus. We plan to investigate the translational, rotational, and vibrational distributions of species produced in the predissociation of van der Waals molecules containing benzene. We will excite the vdW molecule at $248 \mathrm{~nm}$ and probe the product state distribution. This work should begin sometime this summer.

In other work, we have underway a calculational effort to determine the quantitative effects of spontaneous infrared emission on the stabilization of excited adducts formed in radical-radical recombinatin reactions. In conventional thermal unimolecular rate theory, which is based on the Lindemann mechanism, two radicals recombine to produce an excited adduct, whcih can redissociate, or be stabilized by collisions. Emission of infrared radiation also can stabilize the adduct, however, even in the absence of collisions. This process has been shown by R. C. Dunbar to be important in ion-molecule reactions, and we are using detailed master equation calculations to investigate the effects on reactions involving neutrals. For the formation of benzene from the recombination of $\mathrm{H}$-atoms and phenyl radicals, the calculations indicate that at temperatures lower than $400-500 \mathrm{~K}$, collisions are not needed to stabilize the excited benzene. In other words, the low pressure limit equals $k_{\infty}$ and there is no pressure fall-off. This result may have interesting ramifications in laboratory rate constant measurements and in cool, low pressure environments, such as at high altitudes in planetary atmospheres, in interstellar space, and in molecular beam experiments. This work is still underway and we are not sure, at this time, where it will lead. 


\section{Scientific Personnel}

Dr. John R. Barker, Principal Investigator

Ms. Isabelle Cherchneff, Graduate Student (Received Ph.D. in May 1991)

Mr. Jichun Shi, Graduate Student (Received Ph.D. in May 1991)

Mr. Jerrell D. Brenner, Graduate Student

Mr. Alexander Chimbayo, Graduate Student

\section{Publications}

"Vibrational Relaxation of Highly Excited Toluene," Beatriz M. Toselli, Jerrell D. Brenner, Murthy L. Yerram, William E. Chin, Keith D. King, and John R. Barker, J. Chem. Phys., 95, 176 (1991).

"Polycyclic Aromatic Hydrocarbon Optical Properties and Contribution to the Acceleration of Stellar Outflows," Isabelle Cherchneff, John R. Barker, and Alexander G. G. M. Tielens, Astrophys. J., 377, 541-552 (1991).

"Excitation of $\mathrm{CO}_{2}$ by energy transfer from highly vibrationally excited benzene derivatives," Beatriz M. Toselli and John R. Barker, J. Chem. Phys., 95, 8108 (1991).

"Infrared Emission Spectra of Benzene and Naphthalene: Implications for the Interstellar PAH Hypothesis," Jerrell D. Brenner and John R. Barker, Astrophys. J. (Letters), in press (accepted for publication).

"Polycyclic Aromatic Hydrocarbons and Molecular Equilibria in Carbon Rich Stars," Isabelle Cherchneff, John R. Barker, and Alexander G. G. M. Tielens, Astrophy's. J., submitted.

"Polycyclic Aromatic Hydrocarbon Formation in Carbon Rich Stellar Envelopes," Isabelle Cherchneff, John R. Barker, and Alexander G. G. M. Tielens, Astrophys. J., submitted.

"Isotope Effects in the Vibrational Deactivation of Large Molecules," Beatriz M. Toselli and John R. Barker, J. Chem. Phys., in preparation.

\section{Ph.D. 'Dissertations}

Isabelle Cherchneff, "Polycyclic Aromatic Hydrocarbon Radiative Properties and Formation in Carbon-Rich Stellar Outflows," Ph.D dissertation, University of Michigan, Ann Arbor, 1991.

Jichun Shi, "Photochemical kinetics of excited states of $\mathrm{O}_{3}$ and $\mathrm{O}_{2}$; Laboratory measurements and atmospheric implications," Ph.D dissertation, University of Michigan, Ann Arbor, 1991.

\section{DISCLAIMER}

This report was prepared as an account of work sponsored by an agency of the United States Government. Neither the United States Government nor any agency thereof, nor any of their employees, makes any warranty, express or implied, or assumes any legal liability or responsibility for the accuracy, completeness, or usefulness of any information, apparatus, product, or process disclosed, or represents that its use would not infringe privately owned rights. Reference herein to any specific commercial product, process, or service by trade name, trademark, manufacturer, or otherwise does not necessarily constitute or imply its endorsement, recommendation, or favoring by the United $:$ sates Government or any agency thercof. The views mendation, or favoring by the United : sates Government or any age United States Government or any agency thereof. 

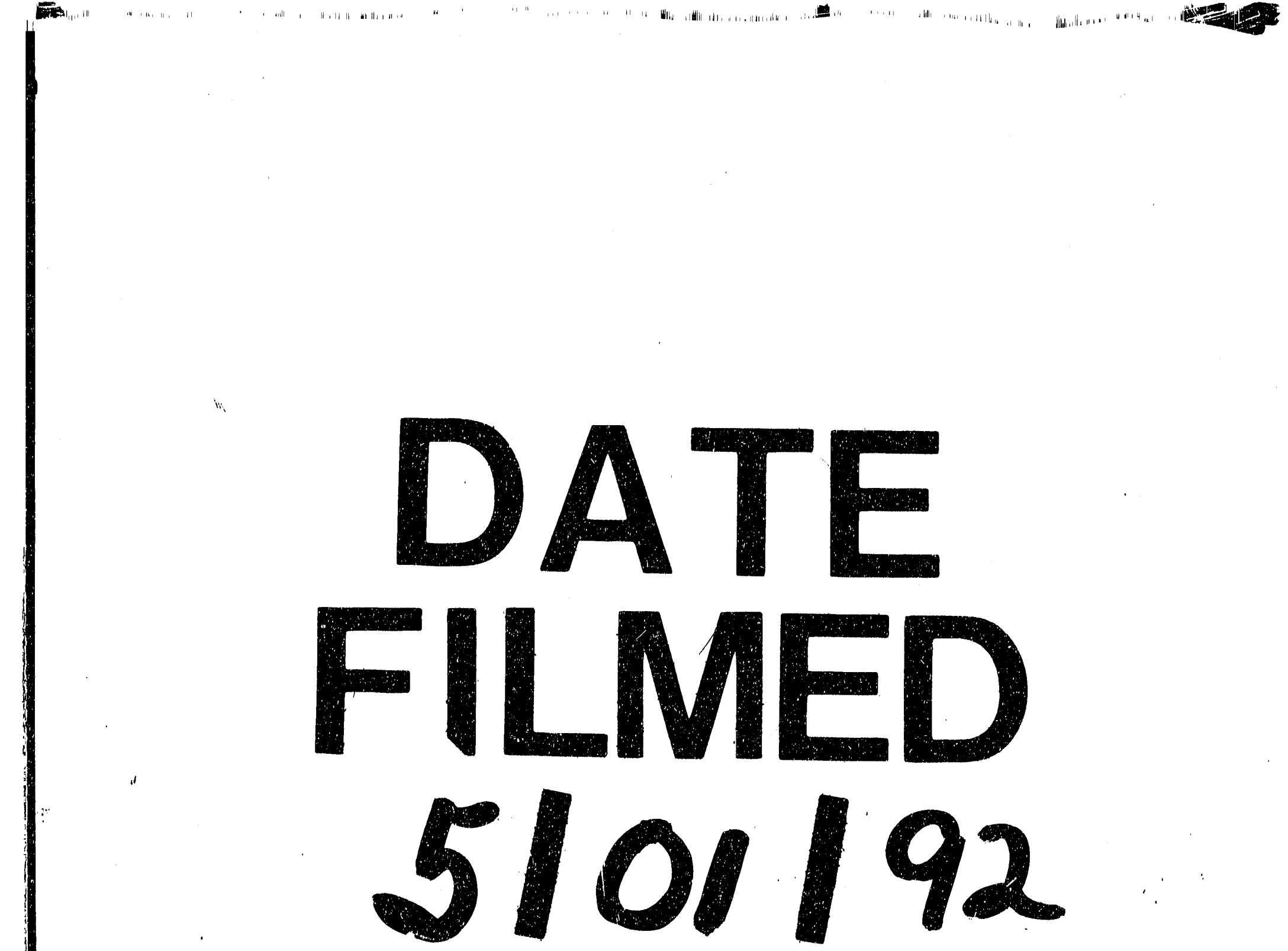


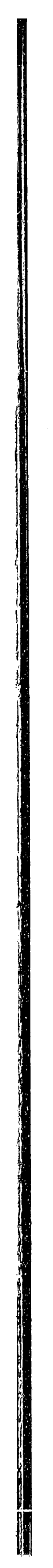

